\title{
Neural Network Identification of a Racing Car Tire Model
}

\author{
Jianfeng Wang $\mathbb{D},{ }^{1}$ Yiqun Liu $\left(\mathbb{D},{ }^{1}\right.$ Liang Ding $\left(\mathbb{D},{ }^{2}\right.$ Jun Li, ${ }^{2}$ Haibo Gao, ${ }^{2}$ \\ Yuhan Liang, and Tianyao Sun ${ }^{1}$ \\ ${ }^{1}$ School of Automotive Engineering, Harbin Institute of Technology, Weihai, Shandong 264209, China \\ ${ }^{2}$ State Key Laboratory of Robotics and System, Harbin Institute of Technology, Harbin 150001, China \\ Correspondence should be addressed to Yiqun Liu; lyq.new@163.com and Liang Ding; liangding@hit.edu.cn
}

Received 3 August 2017; Accepted 16 April 2018; Published 29 May 2018

Academic Editor: Kamran Iqbal

Copyright (C) 2018 Jianfeng Wang et al. This is an open access article distributed under the Creative Commons Attribution License, which permits unrestricted use, distribution, and reproduction in any medium, provided the original work is properly cited.

\begin{abstract}
In order to meet the demands of small race car dynamics simulation, a new method of parameter identification in the Magic Formula tire model is presented in this work, based on an analysis of the Magic Formula tire model structure. A high-precision tire model used for vehicle dynamics simulation is established via this method. It is difficult for students to build a high-precision tire model because of the complexity of widely used tire models such as Magic Formula and UniTire. At a pure side slip condition, building a lateral force model is an example, which illustrate the utilization of a multilayer feed-forward neural network to build an intelligent tire model conveniently. In order to fully understand the difference between the two models, a two-degrees-of-freedom (2 DOF) vehicle model is established. The advantages, disadvantages, and applicable scope of the two tire models are discussed after comparing the simulation results of the 2 DOF model with the Magic Formula and intelligent tire model.
\end{abstract}

\section{Introduction}

The Formula Society of Automotive Engineers (FSAE) race car's higher speed and greater cornering acceleration make tire characteristics significantly influence the vehicle's performance [1-3]. In order to ensure the results of vehicle dynamics simulation are consistent with real vehicle tests, the tire model problem must be solved. It is necessary to build a mathematical model that can accurately express the tire force and moment characteristics [4].

In 1954, Fiala conducted an analysis and performed various experiments pertaining to cornering characteristics and proposed a dimensionless formula of tire characteristics based on a simplified analytical tire model [5]. Since then, the relationship between lateral force/aligning moment and side slip angle has been found.

In 1986, Shape proposed a multispoke model, and its central feature is that the carcass flexibility is associated entirely with independently acting radial spokes. Each spoke is radially and laterally flexible [6]. In 1991, Gim built a complete tire force characteristic formula based on three elementary springs, whose axes of symmetry are perpendicular to each other. This tire model is called the UA-Tire model [79].

In the early 1960s, Pacejka simplified the carcass deformation as a stretched "string", and conducted extensive theoretical and experimental research pertaining to the mechanical characteristics of tires [10]. In 1987, Pacejka and Bakker established a semiempirical tire model that can accurately calculate the lateral force and aligning moment, which is one of the findings of the cooperation project between TU Delft and Volvo [11]. This model was improved in 1989, 1991, 1997, and 2002. Presently, the pneumatic trail $t$ is expressed by a cosine function, and aligning moment can be obtained by multiplying the lateral force $F_{y}$ with the pneumatic trail $t$ and adding the usually small residual moment $M_{z r}$. This further improved tire model is called the Magic Formula tire model [12].

In 1986, Guo proposed a pure lateral slip tire model. The contact pressure distribution was assumed to be uniform in the lateral direction and of arbitrary form in the vertical direction [13]. Later, a combined slip semiempirical tire model was developed. On the basis of various tests and theoretical analyses, a nonlinear and non-steady state tire 
model was proposed. The model can accurately express the six-dimensional wheel force under complex tire motion inputs involving side slip, longitudinal slip, turn slip, and camber [14-16]. Presently, the UniTire model is widely used in research on vehicle handling dynamics.

The intelligent tire model is built on the basis of a neural network and has multi-input, multioutput, and self-adaptive abilities [17]. In 1993, Palkovics and El Gindy applied neural network theory to model tire characteristics for the first time $[18,19]$. Since then, many scholars have begun to research this high-precision and convenient intelligent tire modeling method.

In this paper, the FSAE race car of the Harbin Institute of Technology Racing Team (HRT) is taken as a research object. Firstly, a multiple-step parameter identification method is proposed, based on the analysis of the characteristics of the Magic Formula tire model structure. According to this method, all coefficients of Pacejka 2002 [12] have been obtained after fitting all the Hoosier 18x6R25B tire data at $14 \mathrm{psi}$. Then, the same pure cornering condition test data is fitted using a three-layer feed-forward neural network. In this manner, a simplified intelligent tire model is built. After simulating a nonlinear two-degrees-of-freedom (2 DOF) vehicle model with the Magic Formula and intelligent tire model, the yaw rate at the stepwise input is obtained. Finally, the differences between the two models are analyzed.

\section{Experimental Study}

The FSAE Tire Test Consortium (FSAE TTC) was established to provide high-quality tire data to the participating FSAE teams and was used in the design and setup of race cars [20]. Currently, tire testing work has been carried out five times at Calspan's Tire Research Facility, and the data has been distributed to all the consortium members.

2.1. Tire Characteristic Test Bed. This machine at the Calspan Tire Research Facility (TIRF) is tested by TTC [21]. The tire, wheel, force sensing balance, and hydraulic motor to drive or break the tire are mounted in the movable upper head. The head provides steer, camber, and vertical motions to the tire. These motions (as well as vertical loading) are servo controlled and programmed for maximizing the test efficiency. The 28 -inch wide roadway comprises a stainless steel belt covered with a material that simulates the frictional properties of actual road surfaces. The roadway is driven by one of the two 67-inch diameter drums over which the tire is run. The road speed is servo controlled, and it may be programmed to be constant or varied.

2.2. Experimental Results. The frequency of test data collection is $50 \mathrm{~Hz}$, and the data channels available are elapsed time for the test (ET), road speed $(V)$, slip angle $(\alpha)$, inclination angle $(\gamma)$, slip ratio $(\kappa)$, normal load $\left(F_{z}\right)$, lateral force $\left(F_{y}\right)$, longitudinal force $\left(F_{x}\right)$, aligning moment $\left(M_{z}\right)$, overturning moment $\left(M_{x}\right)$, loaded radius (RL), effective radius (RE), wheel rotational speed $(\mathrm{N})$, road surface temperature (RST),

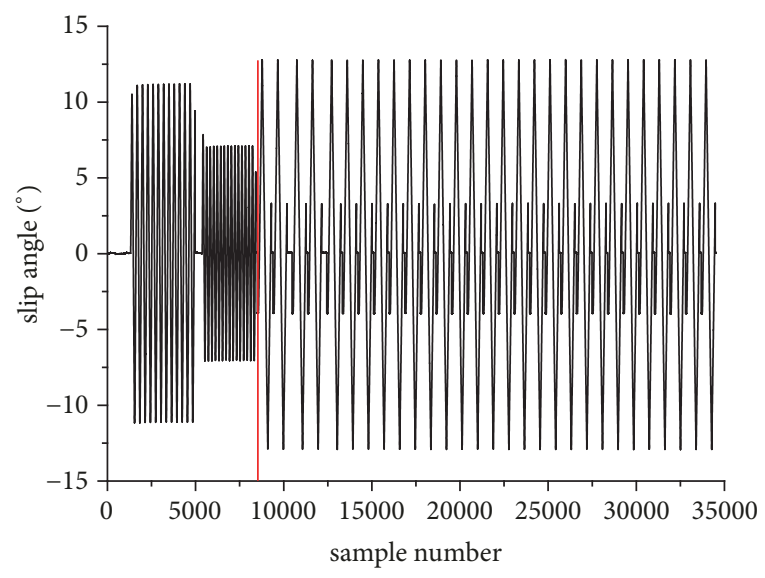

FIGURE 1: Relationship between slip angle and sample number.

ambient room temperature (AMBTMP), and tire surface temperature (TST) [22].

The test data of the Hoosier 18.0x6-10R25B at 14psi is analyzed in this study. The relationship between slip angle and sample number is pictured in Figure 1. The left side of the red line is the process of testing tire stiffness and warming the tire, and this data should be removed when fitting. The right side of the red line is useful test data. The force and moment will be shown in later sections.

\section{Magic Formula Tire Model}

3.1. Introduction to Pacejka 2002. In order to clarify the relationship between the tire characteristic parameters and the six-dimensional tire force, a right-handed system is necessary. The society of Automotive Engineers (SAE) coordinate system is adopted in this paper [23].

The content of Pacejka 2002 is exceedingly abundant, which is not convenient to display in this paper. Please refer to [12] for details. Here is a brief introduction to the parts related to the parameter identification method. Pacejka 2002 is improved in terms of precision and is transformed to the dimensionless form, which makes it easy to understand and identify the parameters [12]. Define the following dimensionless parameters:

$$
\begin{aligned}
F_{z 0}^{\prime} & =\lambda_{F z 0} F_{z 0} \\
d f_{z} & =\frac{F_{z}-F_{z 0}^{\prime}}{F_{z 0}^{\prime}} \\
\alpha^{*} & =\tan \alpha \\
\gamma^{*} & =\sin \gamma .
\end{aligned}
$$

From (1) to (3), it can be found that $d f_{z}$ is equal to zero when vertical load $F_{z}$ equals the nominal load $F_{z 0}^{\prime}$. Those coefficients related to $d f_{z}$ have no influence on the Magic Formula and can be set to zero. In a similar way, when $\alpha$ is equal to zero and $\gamma$ is equal to zero, those coefficients related to $\alpha^{*}$ and $\gamma^{*}$ can also be set to zero. This is the basis of the parameter identification proposed in this paper. 


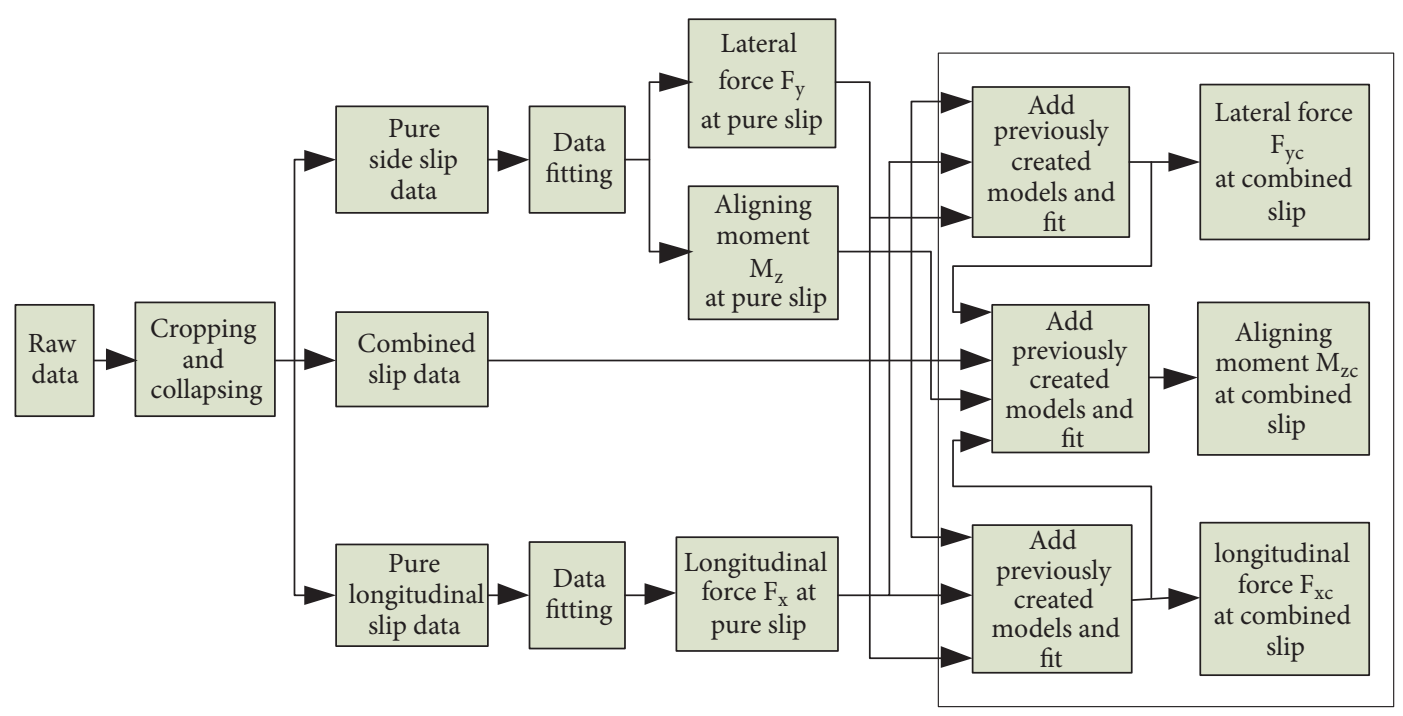

FIGURE 2: General idea of parameter identification.

3.2. Parameter Identification Method. The force and moment formulas at the combined slip of the Magic Formula are based on formulas at pure side slip and longitudinal slip. Parameters of pure side slip and longitudinal slip should be identified before the combined slip. Figure 2 illustrates the general idea of parameter identification. In general, the overall strategy for identification is as follows:

(1) Fit the pure side slip test data and then obtain the lateral force $F_{y}$ at pure side slip.

(2) Fit the pure side slip test data and then obtain the aligning moment $M_{z}$ at pure side slip.

(3) Fit the pure longitudinal slip test data and then obtain the longitudinal force $F_{x}$ at pure longitudinal slip.

(4) On the basis of $F_{y}$ and $F_{x}$, fit the test data at combined slip and then obtain the longitudinal force at combined slip $F_{x c}$.

(5) On the basis of $F_{y}$ and $F_{x}$, fit the test data at combined slip and then obtain the lateral force at combined slip $F_{y c}$.

(6) On the basis of $F_{y c}, F_{x c}$, and $M_{z}$, fit the combined slip test data and then obtain the aligning moment at combined slip $M_{z c}$.

Overturning moment $M_{x}$ and rolling resistance moment $M_{y}$ are not usually used for simulation because of their smaller influence on vehicle handling stability. Thus, only the parameters related to lateral force $F_{y}$, longitudinal force $F_{x}$, and aligning moment $M_{z}$ are identified in this study.

In order to obtain a global optimum solution, a weighting function is applied to the fit. The expression of the objective function is

$$
e=\frac{\sqrt{\sum w_{i}\left(Y-y_{i}\right)^{2}}}{\sum\left|y_{i}\right|},
$$

where $y_{i}$ is the data point value, $Y$ is the corresponding value in the fitted curve, and $w_{i}$ is the weighting function. Typically the values will be 0 or 1 . A value of 0 means the data point $i$ is not considered, and 1 means the opposite.

In the process of fitting the pure slip and combined slip, all coefficients are not fitted together, but step-by-step. Now, the specific fitting process will be introduced by taking the process of fitting the lateral force $F_{y}$ at pure side slip as an example. The program flow chart is shown in Figure 3.

The fitting steps in the program flow chart are as follows:

(1) Input pure side slip raw data and crop and collapse the raw data.

(2) Select the largest or second-largest vertical load as nominal load $F_{z 0}$.

(3) Set the hard boundary of those coefficients unrelated to $d f_{z}$ and $\gamma^{*}$ and set the rest to zero.

(4) Take test data at $\gamma^{*}=0$ and nominal load as fit object.

(5) Fit and obtain the lateral force model $F_{y} \_n d f_{z} n \gamma^{*}$ in which those coefficients related to $d f_{z}$ and $\gamma^{*}$ are zero.

(6) Calculate the least square error $e_{2}$ and check whether $e_{2}$ is less than preset value 2 .

(7) If $e_{2}>$ preset value 2 , change the hard boundary of each coefficient that reached the lower or upper bound and repeat steps (5) to (6); otherwise, proceed to the next step.

(8) Set $90 \%$ and $110 \%$ of $\left.\left.F_{y}\right\lrcorner d f_{z}\right\lrcorner \gamma^{*}$ as the new boundaries.

(9) Set the hard boundary of those coefficients unrelated to $\gamma^{*}$ and set the coefficients related to $d f_{z}$ to zero.

(10) Add test data at other $\gamma^{*}$ and nominal load $F_{z 0}$.

(11) Fit all test data at nominal load and obtain the lateral force model $F_{y} n d f_{z}$. 


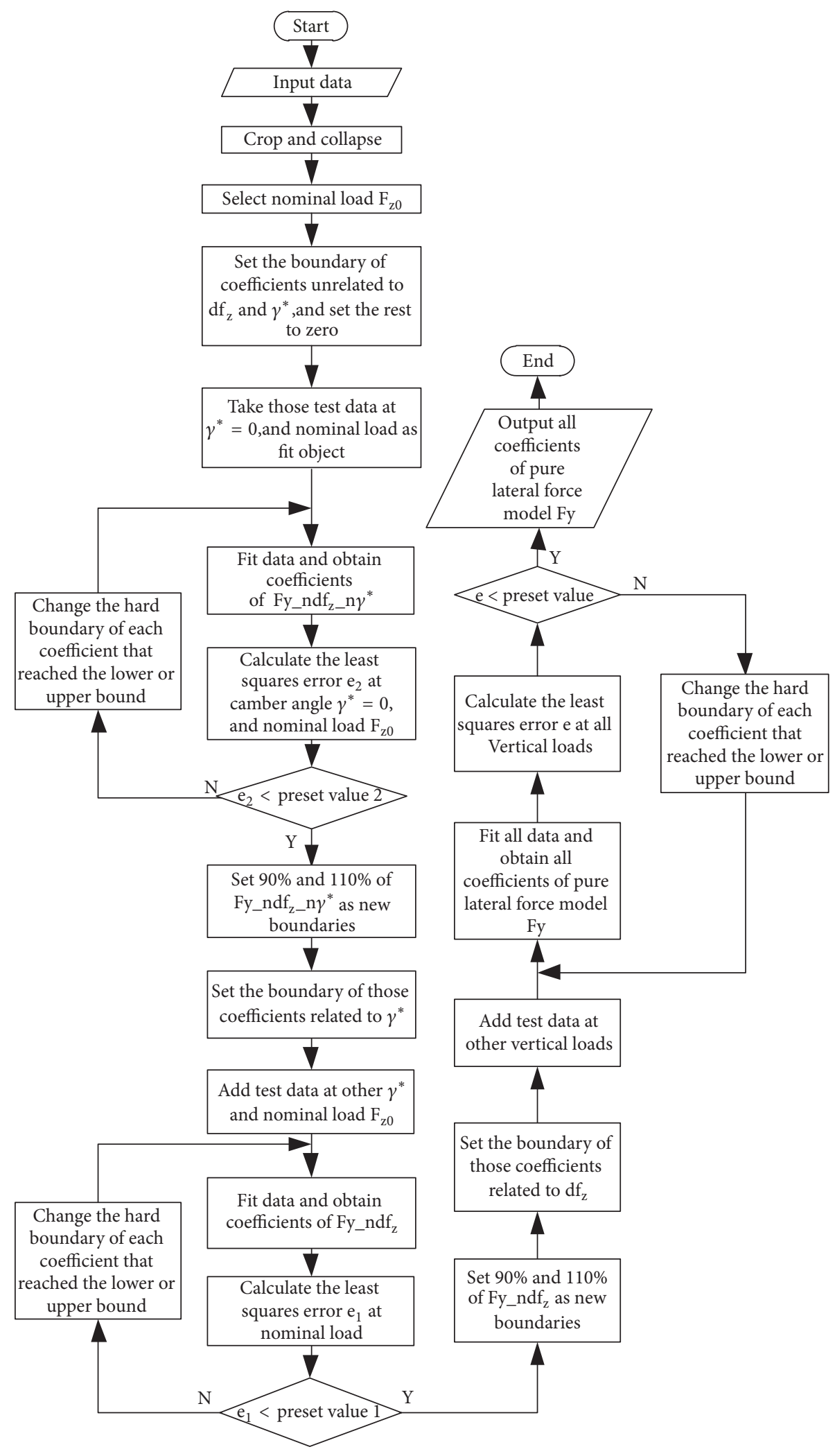

Figure 3: Program flow chart. 


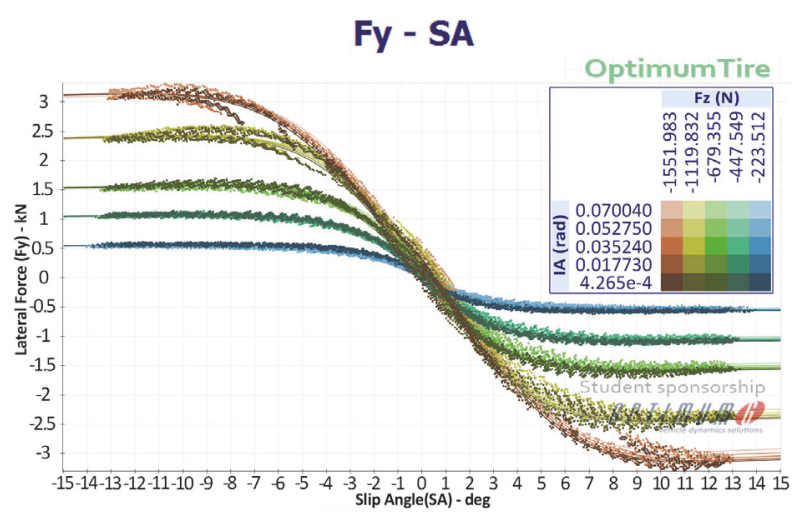

FIGURE 4: Lateral force at pure side slip.

(12) Calculate the least square error $e_{1}$ and check whether $e_{1}$ is less than preset value 1 .

(13) If $e_{1}>$ preset value 1 , change the hard boundary of each coefficient that reached the lower or upper bound and repeat steps (11) to (12); otherwise, proceed to the next step.

(14) Set $90 \%$ and $110 \%$ of $F_{y} n d f_{z}$ as the new boundaries.

(15) Set the hard boundary of those coefficients unrelated to $d f_{z}$.

(16) Add test data at another vertical load.

(17) Fit all test data and obtain the lateral force model $F_{y}$.

(18) Calculate the least square error $e$ and check whether $e$ is less than preset value.

(19) If $e>$ preset value, change the hard boundary of each coefficient that reached the lower or upper bound and repeat steps (17) to (18); otherwise, proceed to the next step.

(20) Output all coefficients of $F_{y}$.

3.3. Parameter Identification Results. Based on the general idea of parameter identification and step-by-step fit strategy, raw data can be processed by MATLAB or Optimum Tire [24]. The latter is adopted in this study. Figures $4-7$ are parameter identification results of the pure side slip condition, pure longitudinal slip condition, and combined slip condition.

The diameter of the test tire, 18 inches, appears smaller compared with the size of the test equipment, which causes the test data points' dispersion. As shown in Figures 4 and 5 , test points for the largest vertical load are not as much as for other loads. This is because outliers are deleted in order to achieve a better fitting result. Some outliers exist in Figure 5 when the slip angle is verified in $-13^{\circ} \sim-12^{\circ}$ and $+12^{\circ} \sim$ $+13^{\circ}$, but the fitting results are still perfect. This is because the weighting function is set to zero in these two areas.

In Figure 6, data points are relatively concentrated when the slip ratio is negative, but it is the opposite when the slip ratio is positive. This is because the braking condition is easier to control and measure compared with the driving condition.

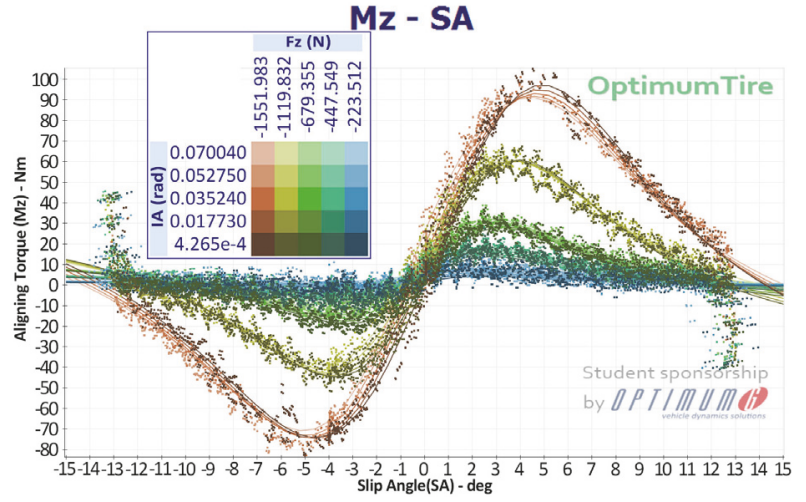

FIgURE 5: Aligning moment at pure side slip.

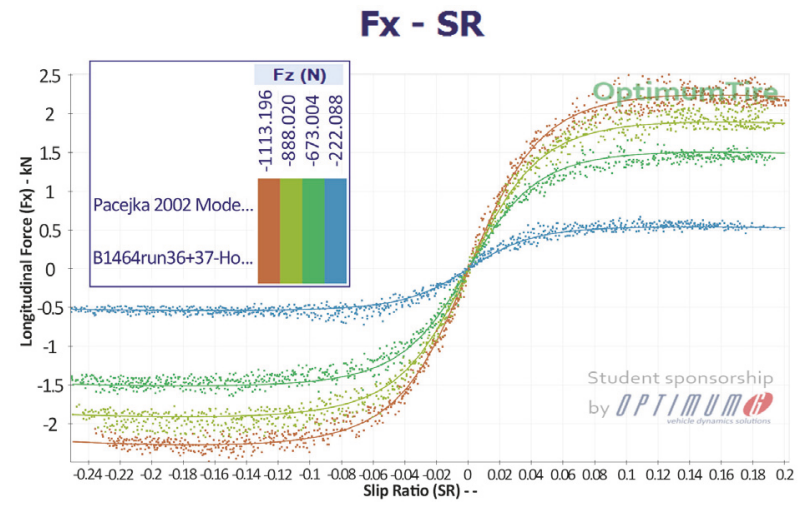

FIgURE 6: Longitudinal force at pure longitudinal slip.

The influence of camber angle on longitudinal force is not considered, so only one fitting curve exists under each vertical load.

The friction ellipse is used to test the global fitting effect. Multidimensional vector space formed by the mapping between the input and output is relatively complex, so most of the fitting process will converge to a local minimum point. If the Magic Formula fitting process converges to a local minimum value, there will be a situation where the fitting results of pure side slip and longitudinal slip condition are better. But an ellipse cannot be obtained in the friction ellipse figure at the combined slip condition. As can be seen from Figure 7, the fitting result is well satisfied, and residual error nearly converges to the global minimum point.

\section{Neural Network Intelligent Tire Model}

Magic Formula is a series of composite functions composed of trigonometric and inverse trigonometric functions, which makes it very difficult to identify parameters. Even more difficult is setting the initial value, because the commonly used numerical optimization method is sensitive to the initial value. Rich experience in engineering is needed to properly estimate the initial value range of each parameter. Therefore, it is necessary to find a simpler method of tire modeling. Since Palkovics et al. applied neural network theory to tire 


\section{Friction Ellipse}

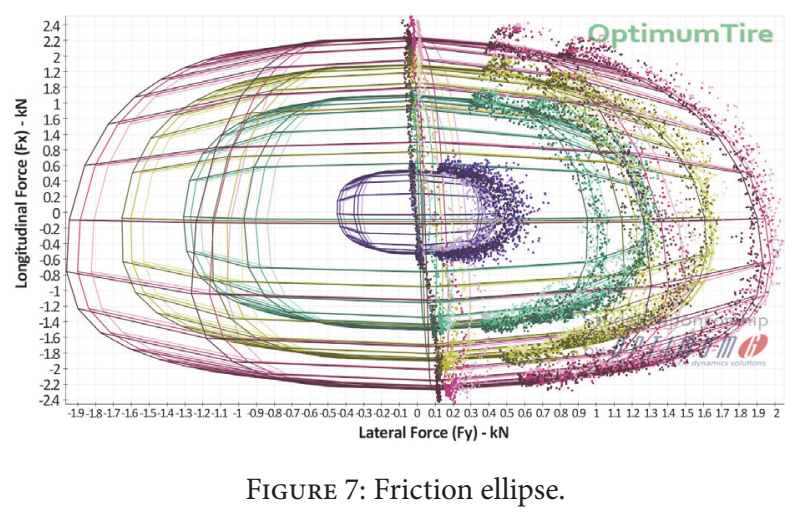

modeling for the first time [18], it has been often applied to engineering practice and achieved good results. The Magic Formula tire model and neural network intelligent tire model depend on the test data of the tire. So, these two models should be classified into the empirical model. Compared to Magic Formula tire model, the shape of the curve fitted by the neural network intelligent model entirely depends on the shape of the test data, but the fitting accuracy is higher and the modeling process is simpler and more convenient. The neural network intelligent tire model is a good choice for vehicle system dynamic simulation.

4.1. Back Propagation Network and Its Improvement. The multilayer feed-forward network is one of the most widely used neural networks. The back propagation (BP) network is a kind of multilayer feed-forward network, trained by the back propagation algorithm. It can be proved that a three-layer feed-forward neural network can simulate any complicated nonlinear system [25]. The BP network has one or more hidden layers. Usually, the sigmoid function is used in a hidden layer, and the purel in function is used in the output layer. Algorithm [26] is adopted to improve the convergence performance of the BP algorithm. Assuming that the weighting function vector of a layer is $w=\left[\mathrm{w}_{1}, \mathrm{w}_{2}, \ldots, \mathrm{w}_{\mathrm{n}}\right]^{\mathrm{T}}$, the adjustment of weighting function $w$ can be defined as follows:

$$
\begin{aligned}
& w^{(k+1)} \\
& =w^{(\mathrm{k})} \\
& \quad-\eta_{k}\left[J^{T}\left(\mathrm{w}^{(\mathrm{k})}\right) J\left(\mathrm{w}^{(\mathrm{k})}\right)+\mu_{\mathrm{k}} I\right] J^{T}\left(\mathrm{w}^{(\mathrm{k})}\right) E\left(\mathrm{w}^{(\mathrm{k})}\right)
\end{aligned}
$$

4.2. Establishing the Neural Network Intelligence Model. MATLAB is one of the most convenient programmable tools to train a BP neural network. Users can use the $M$ language to write a program to train a neural network or use the graphical user interface to train a BP neural network.

Because road speed $V$ is $25 \mathrm{mph}$ throughout the testing process, the influence of driving speed on tire mechanical characteristics cannot be considered. Only the effects of vertical load, slip angle, and camber angle are considered in this intelligent tire model.

Neural network mapping is an expression of the relationship between slip angle, camber angle, vertical load, and lateral force. It is

$$
\text { net }=\left\{F_{y}\right\}=N N\left\{\begin{array}{c}
\alpha \\
\gamma \\
F_{z}
\end{array}\right\} .
$$

The number of neurons in the hidden layer of the network is determined to be 12 by empirical formula [21]. Many time trial results show that the BP neural network has a better convergence rate when the number of hidden layers is 12 . The neurons in two adjacent layers are fully connected, and there is no connection between the neurons in the same layer. The tan-sigmoid function is adopted in the hidden layer, and the purel in function is used in the output layer. Figure 8 shows the structure of the adopted neural network. The LevenbergMarquardt algorithm is chosen as the training algorithm, and mean square error is used to calculate the output error.

The number of test data samples is 27442 . And $70 \%$ of them are randomly selected for training the neural network, $15 \%$ for validation, and $15 \%$ for testing.

After training, if errors distribute mostly near the zero line in the error histogram, the training process should be stopped. Otherwise, the sample proportion between training, validation, and testing, or the number of hidden neurons, should be changed for further training until the error distribution is satisfied. Then, the training results such as the network structure, network input, target output, network output, and error distribution are loaded into the MATLAB workspace, and the Simulink diagram is generated for Simulink simulation. Figure 9 shows the comparison result between the test data and output of the neural network. It can be observed that neural network output agrees well with test data, which illustrates the effectiveness of this training method.

\section{Simulation of Vehicle Handling Stability Based on Tire Model}

5.1. Vehicle Model. Vehicle model is the basis of vehicle dynamics research. It should not only be able to accurately and objectively describe the vehicle actual physical system but also be simplified as much as possible in order to calculate easily. The right model for vehicle dynamics analysis should be appropriately selected to include the dynamic motions of the vehicle that need to be studied. The focus of this study is the tire model. In order to be able to make the model accurate and easy to analyze, a 2-DOF vehicle model is adopted. The steering systems, suspension, and pitch and roll motion of vehicle are ignored in this model. In addition, the absolute speed of the vehicle remains unchanged. Assumptions are as follows:

(1) Ignore the influence of the driving force on the tire cornering property.

(2) Ignore the air force. 


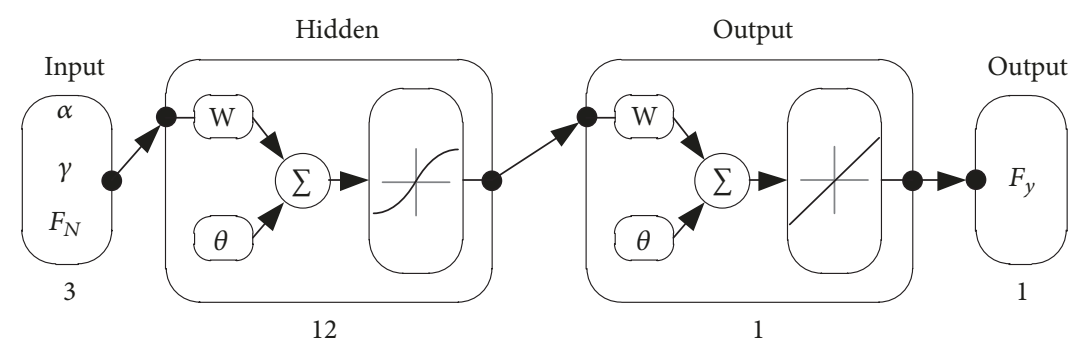

FIGURE 8: Structure of adopted neural network.

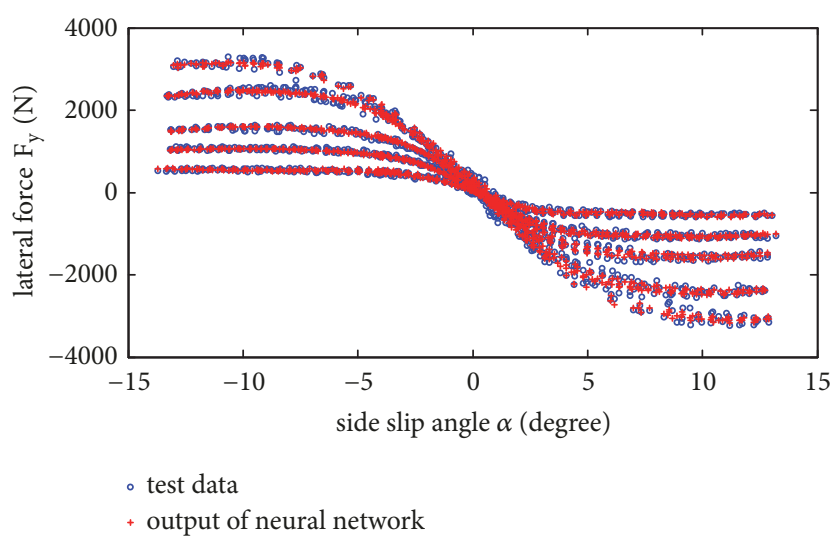

Figure 9: Comparisons between test data and output of the neural network.

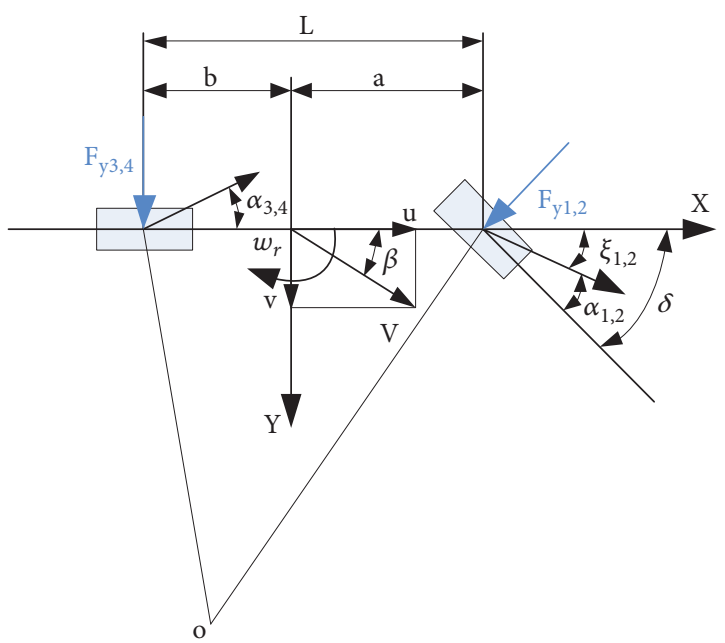

FIGURE 10: Vehicle motion and force analysis diagram.

(3) Ignore wheel load transfer.

(4) Ignore the influence of aligning moment.

In this way, the vehicle model is simplified as a motorcycle model. Figure 10 is the vehicle motion and force analysis diagram. The model consists of two elastic tires. And the model has two DOF, translational motion of vehicle in lateral direction and rotational motion of yaw.
According to the laws of dynamics for rigid bodies and the SAE coordinate system [23], the governing equations of motion of the vehicle model have been derived.

$$
\begin{aligned}
F_{Y 1}+F_{Y 2}+F_{Y 3}+F_{Y 4} & =m\left(\dot{v}+u w_{r}\right) \\
\left(F_{Y 1}+F_{Y 2}\right) \cdot a-\left(F_{Y 3}+F_{Y 4}\right) \cdot b & =I_{Z} \dot{w}_{r},
\end{aligned}
$$

where $u, v$, and $w_{r}$ are vehicle longitudinal speed, lateral speed, and the yaw rate, respectively, $m$ is mass of the vehicle, and $I_{Z}$ is the vehicle inertia moment about $\mathrm{Z}$-axis.

Lateral force is closely related to tire slip angle, and the slip angle can be expressed as a function of the motion parameters of vehicle. In Figure 11, $\alpha_{1}$ and $\alpha_{2}$ are the slip angle of left front tire and right front tire, respectively. $\alpha_{3}$ and $\alpha_{4}$ are the slip angle of left rear tire and right rear tire, respectively. Sideslip angle is $\beta=v / u$. $\xi$ is the angle between the tire speed and the $\mathrm{X}$-axis and can be calculated as follows:

$$
\xi=\arctan \frac{v+a w_{r}}{u} \approx \beta+\frac{a w_{r}}{u} .
$$

According to the tire coordinate system and vehicle coordinate system, the slip angle of each tire can be expressed as follows:

$$
\begin{aligned}
& \alpha_{1}=\alpha_{2}=-(\delta-\xi)=\beta+\frac{a w_{r}}{u}-\delta \\
& \alpha_{3}=\alpha_{4}=\frac{v-b w_{r}}{u}=\beta-\frac{b w_{r}}{u} .
\end{aligned}
$$

The term $F_{Y}$ in (8) and (9) is the tire force along the Yaxis. It can be expressed as a function of lateral force $F_{y}$ and tire steering angle $\delta$.

$$
\begin{aligned}
& F_{Y 1}=F_{y 1} \cos \delta \\
& F_{Y 2}=F_{y 2} \cos \delta \\
& F_{Y 3}=F_{y 3} \\
& F_{Y 4}=F_{y 4} .
\end{aligned}
$$

According to previous research on tire characteristics, lateral force $F_{y}$ is a function of vertical load $F_{z}$, slip angle $\alpha$, and camber angle $\gamma$ for each tire:

$$
F_{y}=F_{y 0}\left(\alpha, \gamma, F_{z}\right)
$$


TABLE 1: Specifications of the FSAE race car.

\begin{tabular}{lcc}
\hline Vehicle parameters & Symbol & Value \\
\hline Mass & $\mathrm{m}$ & $230 \mathrm{~kg}$ \\
Sprung mass & $\mathrm{m}_{\mathrm{s}}$ & $199.1 \mathrm{~kg}$ \\
Wheelbase & $\mathrm{L}$ & $1.530 \mathrm{~m}$ \\
Front axle distance from C.G. & $\mathrm{a}$ & $0.795 \mathrm{~m}$ \\
Rear axle distance from C.G. & $\mathrm{b}$ & $0.735 \mathrm{~m}$ \\
Track & $\mathrm{T}$ & $1.140 \mathrm{~m}$ \\
Yaw moment of inertia & $\mathrm{I}_{\mathrm{z}}$ & $70.046 \mathrm{~kg} \cdot \mathrm{m}^{2}$ \\
\hline
\end{tabular}

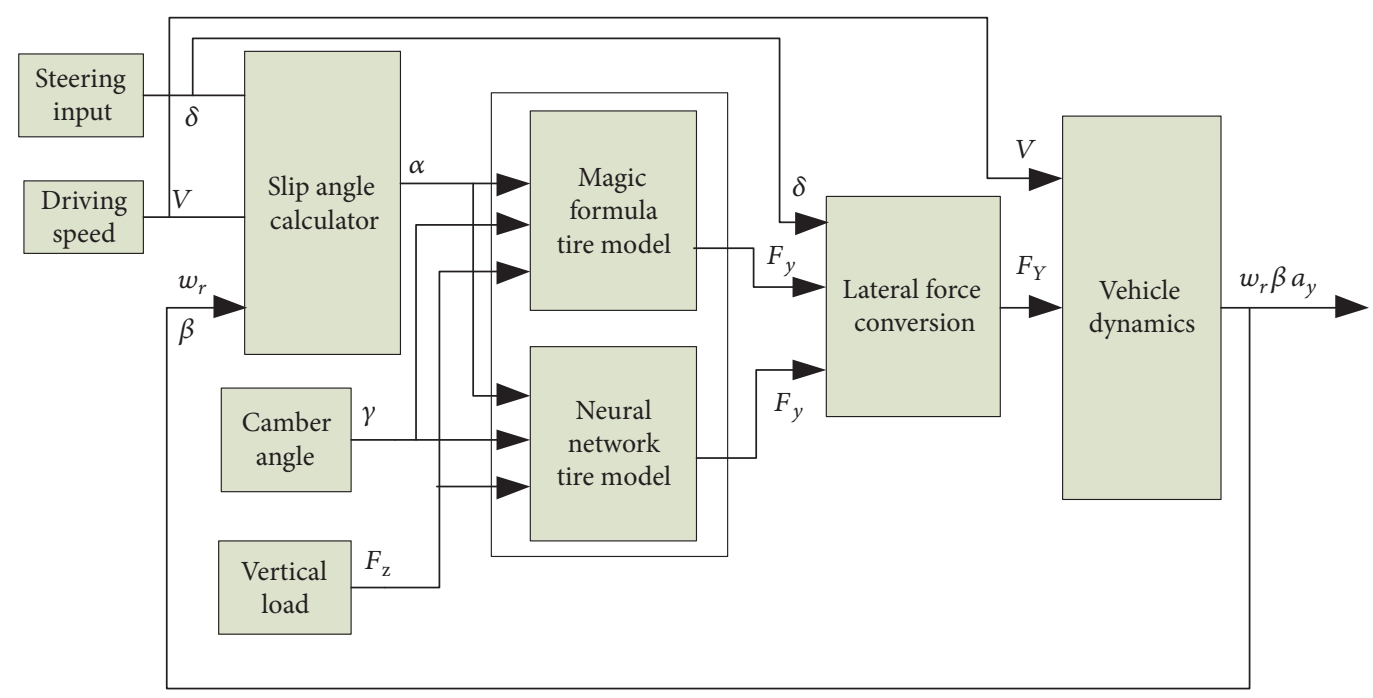

FIGURE 11: Simulation diagram of the nonlinear vehicle model with 2 DOF.

In order to ensure the accuracy, convenience, and easy understanding of the vehicle model, it is divided into six parts: input module, slip angle calculation module, vertical load calculation module, tire module, lateral force conversion module, and governing equations of vehicle motion module. On the basis of the two tire models built in previous sections, the simulation diagram of a nonlinear vehicle model with 2 DOF is established, as shown in Figure 11.

In order to complete the simulation of vehicle dynamic model with different tire models with the stepwise steering input, the specifications of the FSAE race car are listed in Table 1.

5.2. Analysis of Simulation Results. Speed of the HRT race car is designed to be 0 to $100 \mathrm{~km} / \mathrm{h}$. Range of the hand-wheel steering angle is $-120^{\circ} \sim+120^{\circ}$, and range of the tire steering angle is $-30^{\circ} \sim+30^{\circ}$. Therefore, vehicle speeds for simulation are selected as $5 \mathrm{~m} / \mathrm{s}, 15 \mathrm{~m} / \mathrm{s}$, and $25 \mathrm{~m} / \mathrm{s}$. Inputs of the front tire steering angles are $4^{\circ}, 8^{\circ}, 12^{\circ}, 16^{\circ}$, and $20^{\circ}$.

Figures $12(\mathrm{a}), 12(\mathrm{c})$, and $12(\mathrm{e})$ are the yaw rate response when the vehicle speed is $5 \mathrm{~m} / \mathrm{s}, 15 \mathrm{~m} / \mathrm{s}$, and $25 \mathrm{~m} / \mathrm{s}$, respectively. The front tire steering angle is $4^{\circ}, 8^{\circ}, 12^{\circ}, 16^{\circ}$, and $20^{\circ}$ in each vehicle speed. Figures $12(\mathrm{~b}), 12(\mathrm{~d})$, and $12(\mathrm{f})$ are the tire side slip angle variation in three different speed simulation conditions.
As can be seen from Figure 12(a), yaw rate responses of the vehicle with Magic Formula tire model and neural network intelligent tire model can both reach steady states with the front tire steering angle ranging from $4^{\circ}$ to $20^{\circ}$, and the differences are extremely small at low speed. As shown in Figure 12(b), slip angle of tire in the two vehicle models does not exceed the scope of the slip angle in test data $-13^{\circ} \sim+13^{\circ}$.

In Figure 12(c), the yaw rate response of the vehicle with Magic Formula tire model can reach steady state with the front tire steering angle ranging from $4^{\circ}$ to $20^{\circ}$ at medium vehicle speed. The response of vehicle with neural network intelligent tire model can reach steady state with the front tire steering angle ranging from $4^{\circ}$ to $16^{\circ}$, but it tends to keep rising with the front tire steering angle varying around $20^{\circ}$. As shown in Figure 12(d), the solid line standing for the slip angle of the front tire of the vehicle with the Magic Formula tire model exceeds the range $-13^{\circ} \sim+13^{\circ}$. The red and black dashed lines standing for the slip angle of the front tire of the vehicle with the neural network intelligent tire model both exceed the range $-13^{\circ} \sim+13^{\circ}$. From Figures 12 (c) and $12(\mathrm{~d})$, it can be inferred that when the tire steering angle is approximately $20^{\circ}$; the reason the yaw rate of the vehicle with the neural network intelligent tire model keeps rising is that the slip angle exceeds the range $-13^{\circ} \sim+13^{\circ}$.

In Figure 12(e), at high vehicle speed, the yaw rate response of the vehicle with the Magic Formula tire model 


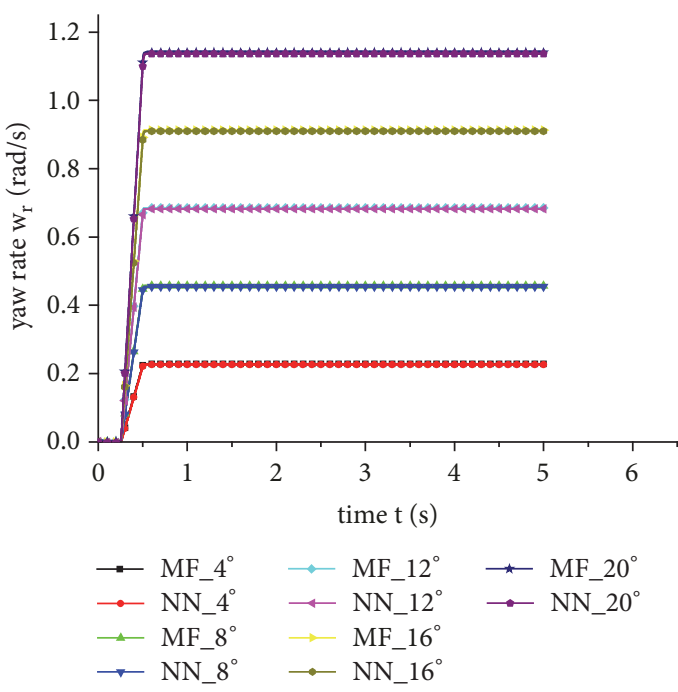

(a) Yaw rate response when speed is $5 \mathrm{~m} / \mathrm{s}$.

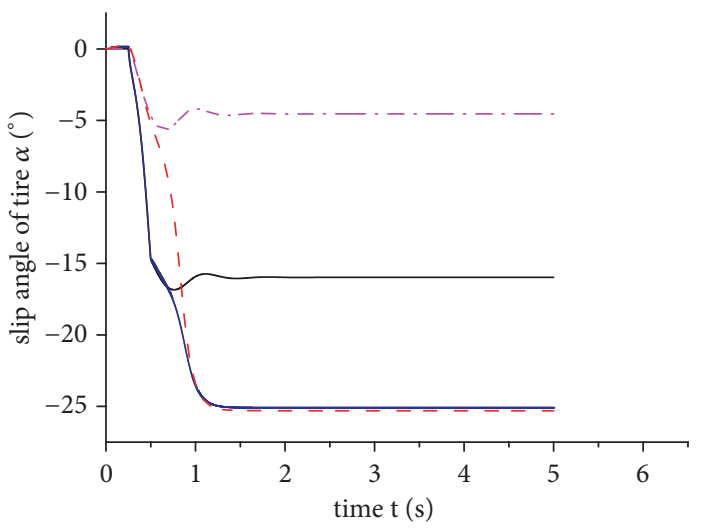

- SA1,2 MF_15_20 … SA1,2 NN_15_20

-.-SA3,4 MF_15_20 - - SA3,4 NN_15_20

(c) Yaw rate response when speed is $15 \mathrm{~m} / \mathrm{s}$.

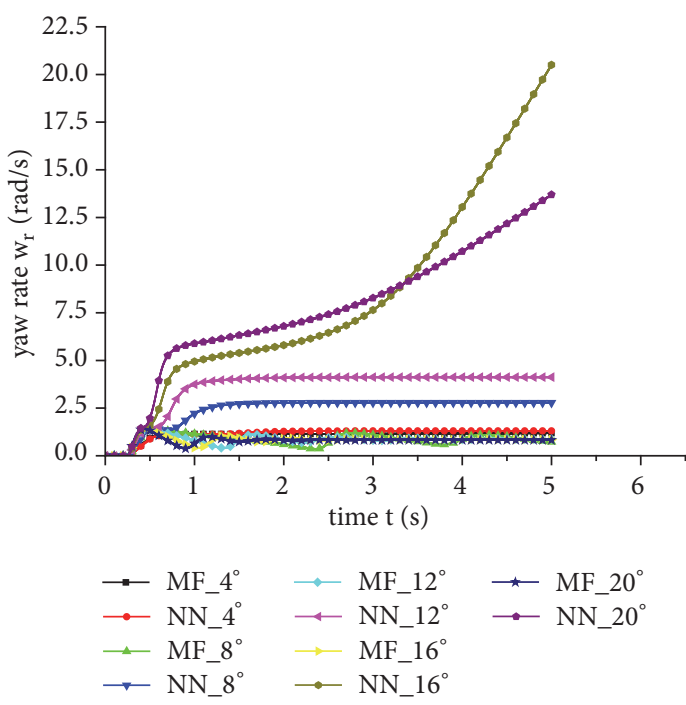

(e) Yaw rate response when speed is $25 \mathrm{~m} / \mathrm{s}$.

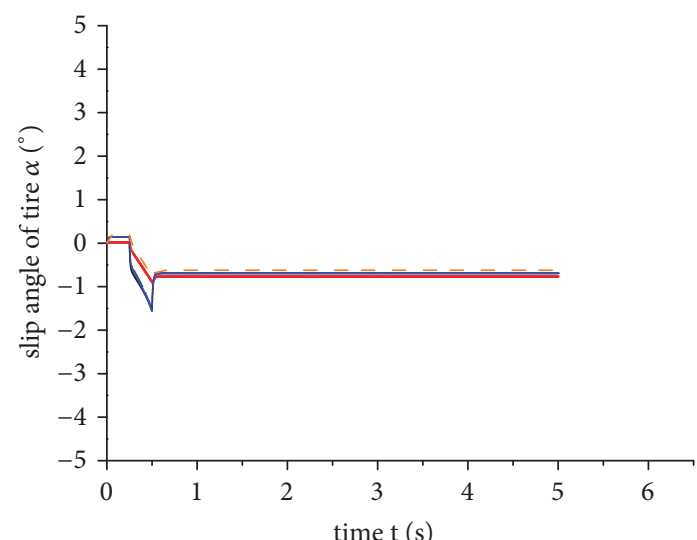

- SA1,2 MF_5_20 -..-SA1,2 NN_5_20

-... SA3,4 MF_5_20 - - SA3,4 NN_5_20

(b) Slip angle when tire steering angle is $20^{\circ}$.

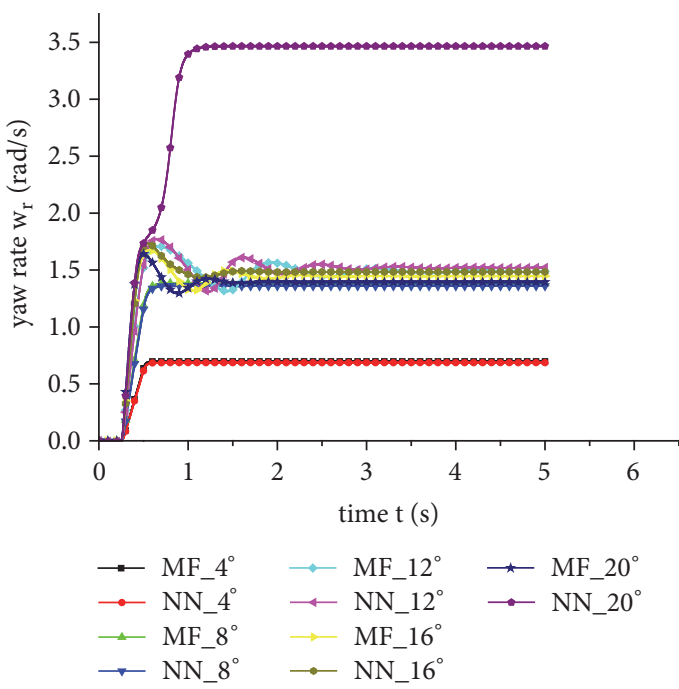

(d) Slip angle when tire steering angle is $20^{\circ}$.
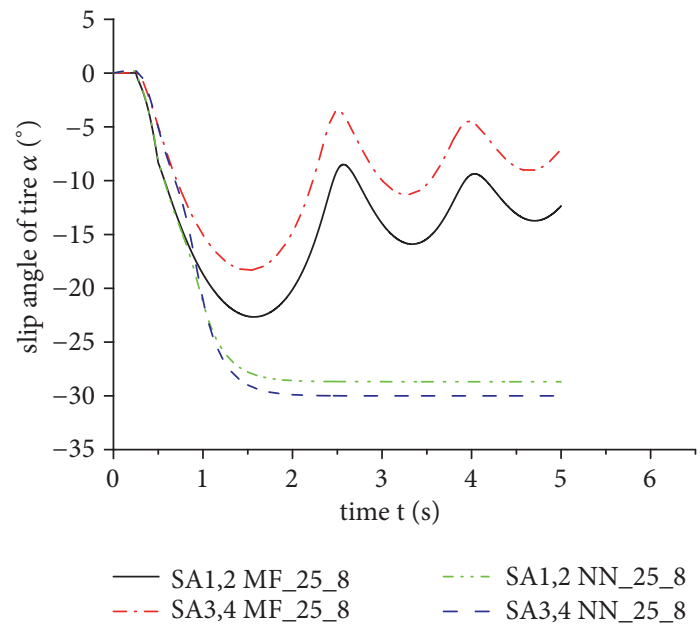

(f) Slip angle when tire steering angle is $8^{\circ}$.

FIgURE 12: The comparison of simulation results. 
can reach steady state with the front tire steering angle ranging from $4^{\circ}$ to $20^{\circ}$, but the response of the vehicle with the neural network intelligent tire model cannot reach steady state when the front tire steering angle exceeds $8^{\circ}$. In Figure 12(f), the slip angle of the tire in both vehicle models exceeds the scope of the slip angle in the test data.

From Figures 12(a)-12(f), when the speed is low,or the speed is high but tire steering angle is low, the vehicle model with Magic Formula tire model or neural network tire model can both correctly predict the motion of the race car. And accuracy of the neural network tire model is higher compared with that of the Magic Formula tire model. When the speed is high or the tire steering angle is too large, the vehicle model with the Magic Formula tire model can still express the car motion state, but the vehicle model with the neural network model cannot predict it. The reason is that the Magic Formula tire model has greater extrapolation ability than the neural network model. However, the neural network intelligent tire model has good interpolation ability, because it is highly dependent on test data.

Before applying the two tire models, the maximum range of the side slip angle of the tire should be estimated according to vehicle speed, steering angle, and other parameters. If the maximum tire side slip angle exceeds the tire side slip angle test range, Magic Formula tire model should be selected. Otherwise, neural network intelligent tire model can be used.

\section{Summary and Conclusion}

According to the structural characteristics of the Magic Formula, an effective method is proposed to identify parameters, namely, the step-by-step fitting method. All coefficients of the Magic Formula are classified into different parts, and all parts are fitted step-by-step. This fitting process is aided by Optimum Tire software. The effectiveness of this method can be verified from the fitting results.

The neural network intelligent tire model for the tire Hoosier 18x6R25B is built on the basis of existing research and test data. It can be inferred that this tire modeling method is more efficient and has higher precision after analyzing the comparison results with the Magic Formula tire model.

After simulating the vehicle model with the Magic Formula tire model and neural network intelligent tire model, and comparing the yaw rate of vehicle, the applicable scope of the two tire models is determined. When the speed is high and the steering angle of the front tire is large, the Magic Formula tire model is more appropriate. When the speed is lower or the steering angle is small, the neural network tire model can be used in the vehicle dynamics simulation.

\section{Conflicts of Interest}

The authors declare that there are no conflicts of interest regarding the publication of this paper.

\section{Acknowledgments}

The authors are grateful to the Calspan Tire Research Facility which supply the tire test as sponsors of HIT racing team. The authors are also grateful to the HRT Formula Student Team. This study was supported in part by the National Natural Science Foundation of China (51705097), the project funded by China Postdoctoral Science Foundation (2017M621258), the project supported by the Scientific Research Foundation of Harbin Institute of Technology at Weihai (HIT(WH)201601), the Foundation of Chinese State Key Laboratory of Robotics and Systems (Grant no. SKLRS201602B), and the "111 Project" (Grant no. B07018).

\section{References}

[1] J. Romero and N. Queipo, "Reliability-based and deterministic design optimization of a FSAE brake pedal: a risk allocation analysis," Structural and Multidisciplinary Optimization, vol. 56, no. 3, pp. 681-695, 2017.

[2] G. Newton, "FSAE competition challenges engineering students," Sound and Vibration, vol. 41, no. 4, pp. 6-10, 2007.

[3] T. Y. Pang and H. Tristian, "Experimental and finite element nonlinear dynamics analysis of formula SAE impact attenuator," Nonlinear Approaches in Engineering Applications 2, vol. 9781461468776, pp. 213-229, 2014.

[4] D. Robertson and G. J. Delagrammatikas, "The suspension system of the 2009 cooper union fsae vehicle: A comprehensive design review," SAE International Journal of Passenger Cars-Mechanical Systems, vol. 3, no. 1, pp. 366-380, 2010.

[5] E. Fiala, "Seitenkräfte am rollenden Luftreifen," VDI Zeitschrift, vol. 96, 1954.

[6] R. S. Sharp, "On the accurate representation of tyre shear forces by a multi-radial-spoke model," Vehicle System Dynamics, vol. 18, pp. 528-541, 1989.

[7] G. Gim and P. E. Nikravesh, "An analytical model of pneumatic tyres for vehicle dynamic simulations. Part 1: pure slips," International Journal of Vehicle Design, vol. 11, no. 6, pp. 589618,1990

[8] G. Gim, "An analytical model of pneumatic tyres for vehicle dynamic simulations. Part 2: comprehensive slips," International Journal of Vehicle Design, vol. 12, pp. 19-39, 1991.

[9] G. Gim and P. E. Nikravesh, "Analytical model of pneumatic tyres for vehicle dynamic simulations. Part 3. Validation against experimental data," International Journal of Vehicle Design, vol. 12, no. 2, pp. 217-228, 1991.

[10] H. B. Pacejka, "Analysis of the Shimmy Phenomenon," Proceedings of the Institution of Mechanical Engineers: Automobile Division, vol. 180, no. 1, pp. 251-268, 2006.

[11] E. Bakker, L. Nyborg, and H. B. Pacejka, "Tyre modelling for use in vehicle dynamics studies," SAE Technical Papers, Article ID 870421, pp. 1-15, 1987.

[12] H. Pacejka, Tire and Vehicle Dynamics, Butterworth-Heinemann, Oxford, 2012.

[13] K. H. Guo, Vehicle Handling Dynamics Principle, Phoenix Science Press, Nanjing, China, 2011.

[14] K. H. Guo and L. Qing, Nonsteady state cornering characteristics medeling of tire, vol. 2, Automot Technol, 1996.

[15] K. Guo, D. Lu, S.-K. Chen, W. C. Lin, and X.-P. Lu, "The UniTire model: a nonlinear and non-steady-state tyre model for vehicle dynamics simulation," Vehicle System Dynamics, vol. 43, supplement 1, pp. 341-358, 2005.

[16] K. Guo and D. Lu, "UniTire: Unified tire model for vehicle dynamic simulation," Vehicle System Dynamics, vol. 45, no. 1, pp. 79-99, 2007. 
[17] M. Gadola, P. Maffezzoni, and D. Cambiaghi, "Tyre modelling with the use of neural nets for vehicle dynamics studies," in Proceedings of the in. International Tourism and Travel Fair. Applied Mechanics and Auto, pp. 57-78, 1994.

[18] L. Palkovics and M. El-Gindy, "Neural network representation of tyre characteristics: the neuro-tyre," International Journal of Vehicle Design, vol. 14, no. 5-6, pp. 563-591, 1993.

[19] M. El-Gindy and L. Palkovics, "Possible application of artificial neural networks to vehicle dynamics and control: a literature review," International Journal of Vehicle Design, vol. 14, no. 5-6, pp. 592-614, 1993.

[20] E. M. Kasprzak and D. Gentz, "The formula sae tire test consortium-tire testing and data handling," SAE Technical Papers, 2006.

[21] K. D. Bird and J. F. Martin, "The Calspan Tire Research Facility: Design, Development, and Initial Test Results," in Proceedings of the National Automobile Engineering Meeting.

[22] E. M. Kasprzak, "Milliken Research Associates.Contents_ round5".

[23] T. D. Gillespie, Fundamentals of Vehicle Dynamics, SAE International, 1992.

[24] "optimumg," http://www.optimumg.com/.

[25] H. Wei, Theory and Method of Neural Network Structure Design, National Defence Industry Press, 2005.

[26] A. A. Suratgar, M. B. Tavakoli, and A. Hoseinabadi, "Modified Levenberg-Marquardt method for neural networks training," in Proceedings of the Wec 05: Fourth World Enformatika Conference, pp. 46-48, tur, June 2005. 




\section{Enfincering}
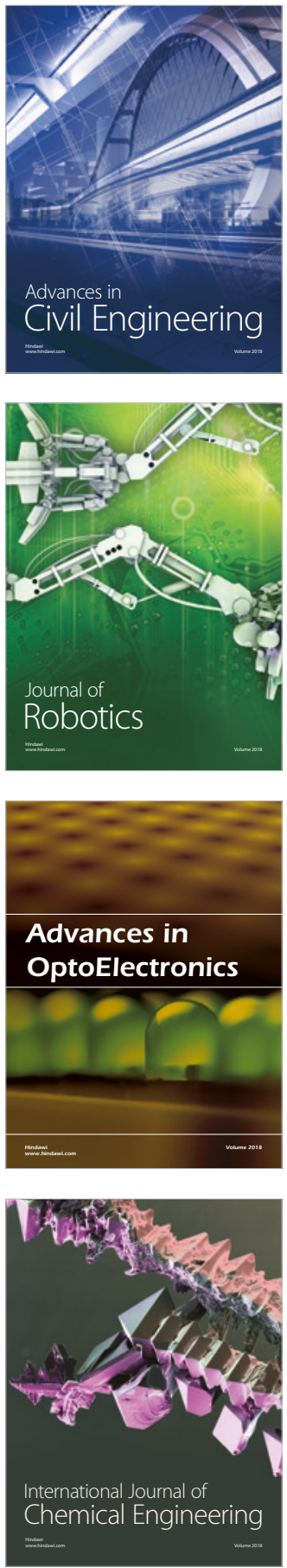

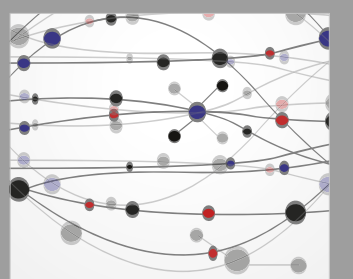

\section{Rotating \\ Machinery}

The Scientific World Journal

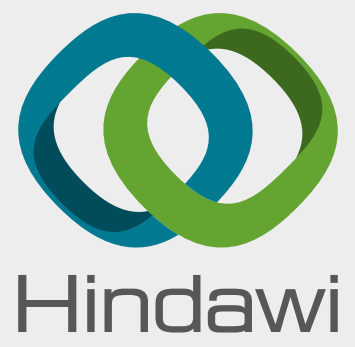

Submit your manuscripts at

www.hindawi.com
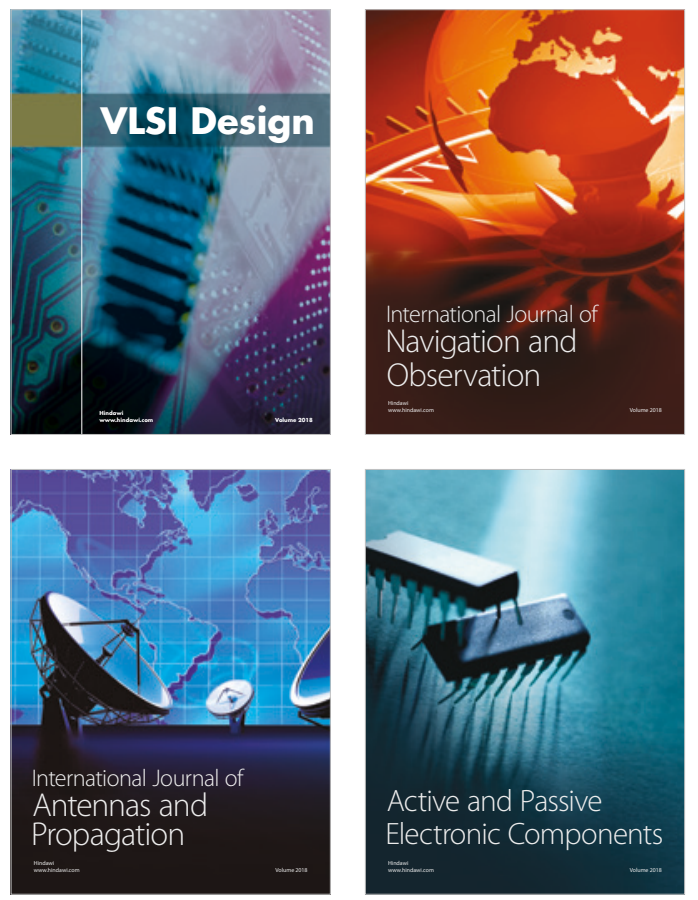
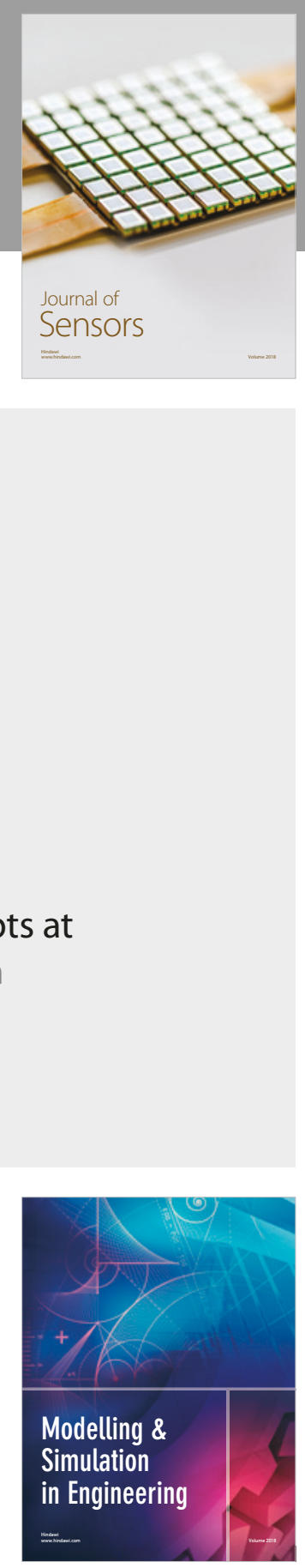

\section{Advances \\ Multimedia}
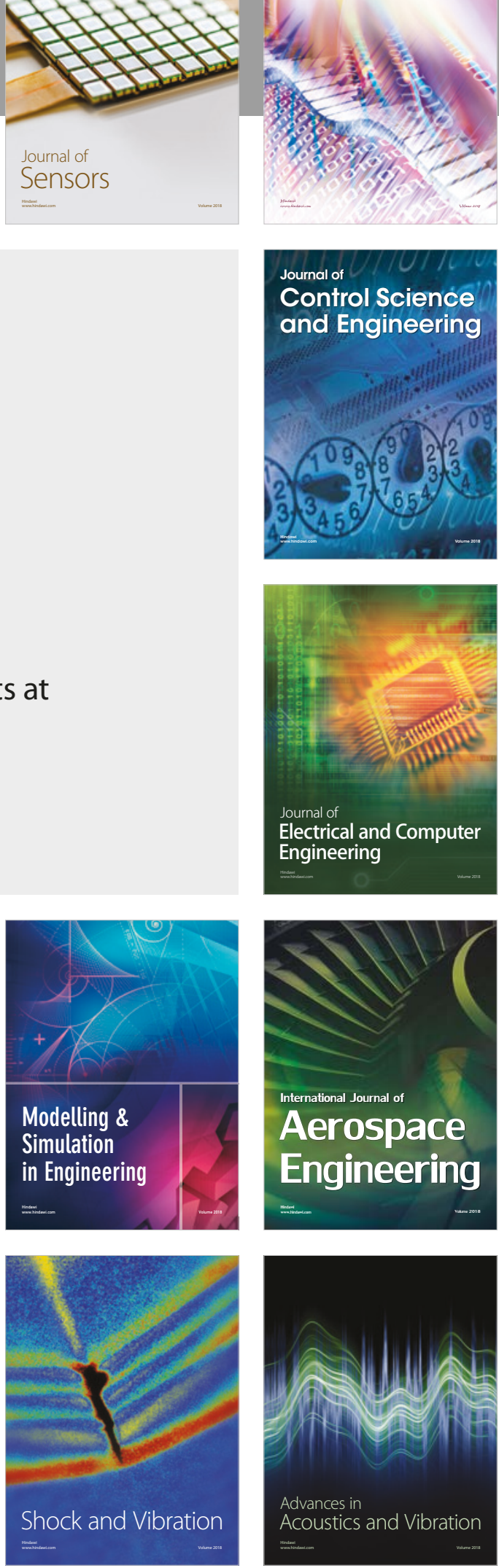